\title{
A New Guide Lifter for the Transceiver of USBL
}

\author{
Chai YONGSHENG, Min XIAOCHEN, Liu MI, Zhang MAOYUAN, Wei SHANGTAO, Wang CHANGHUI*, Liang MEI
}

\begin{abstract}
A new guide lifter has been put forward for the transceiver of Ultra Short Base Line (USBL) with a worm gear reducer applied as self-locking of the lifter and a chain structure applied to drive the sliding shaft moving up and down. The new device is $7500 \mathrm{~mm}$ long and connected to the end of the transceiver. Linear motion products are introduced to ensure the shaft unable to rotate and the position measurements are provided by position sensors. A heavy self-sealing sliding bearing, which is $800 \mathrm{~mm}$ in length, keeps the shaft running reliably. Then the three-dimensional model is built and the structure parameters of the lifter are calculated. Later, the working process of the lifter is simulated to guarantee the movement parameters meet the request of USBL. Finally, the experiment on the intensity and stiffness of the lifter is carried out via the finite element model of the lifter built in ANSYS with the maximum load conditions and the result has been experimentally verified. This device provides a reliable approach of operating USBL which plays a vitally important role in ocean exploration and the research results are successfully applied to the scientific research vessels of Dayang No. 1 as well as Xiangyanghong No. 9.
\end{abstract}

Keywords: design; lifter; simulation; Ultra Short Base Line (USBL) system

\section{INTRODUCTION}

With the rapid development of underwater technology during the last two decades, affordable sensors and underwater vehicles have been widely employed for ocean exploration. The transceiver of the Ultra Short Base Line (USBL) plays a vitally important role in ocean exploration $[1,2]$. The device is the result of the characteristics of the telescopic and the mechanical transmission requires high structural stability $[3,4]$. To improve the precision of USBL, a new guide lifter is put forward for the transceiver. The main aspects of the paper are as follows: first, the machine type is chosen to meet the request of USBL and the structure parameters of the lifter are calculated. The three-dimensional model of the lifter is built for further investigation. Second, the working process of the lifter is simulated to make sure whether the main movement parameters can meet the request of USBL. Third, according to finite element analysis theory, finite element method model of the lifter is built in ANSYS. We adopt obvious dynamics method to analysis the dynamic response of the lifter running on the maximum load conditions. The checkout on the intensity and stiffness of the lifter has been presented.

\section{DESIGN AND ANALYSIS}

\subsection{Transceiver Requirements}

The transceiver diameter $D_{0}=400 \mathrm{~mm}$, height $H_{0}=$ $450 \mathrm{~mm}$, weight $W_{0} \leq 60 \mathrm{~kg}$.

The transceiver is installed at the end of the lifter which can be extended $3000 \mathrm{~mm}$ outside the bottom shell plate.

The cable diameter of transceiver $d_{1} \leq 60 \mathrm{~mm}$.

When the USBL is working, the rolling angle $\varepsilon \leq 15^{\circ}$, rolling period $T=6.5 \mathrm{~s}$. $\mathrm{m} / \mathrm{s})$.

The relative velocity of transceiver $v_{1} \leq 6$ knots $(3.087$

The maximum deflection angle of the lifter is within \pm $0.1^{\circ}$ when the transceiver extends $3000 \mathrm{~mm}$ outside the ship.

Transceiver is driven by motor smoothly and also can be operated by hand when in emergency. The lifting or fall time is less than $60 \mathrm{~s} \mathrm{[5].}$

\subsection{Design Scheme}

In this paper, three structure schemes of the lifter are presented (Fig. 1): hydraulic transmission structure, screw transmission structure and chain transmission structure. The following is the analysis of their advantages and disadvantages:

Hydraulic transmission: The advantage of hydraulic drive is that it can enlarge force and achieve infinitely variable speed as well as smooth transmission. The working parts of the lubrication condition are good and can realize frequent commutation with long operating life. The transfer motion impact is small, easy to achieve overdrive protection. Its liquid medium makes it easy to leak which becomes the crucial drawback of hydraulic drive. Once the fault occurred, the leak point is not easy to find. The inaccuracy of component manufacturing, complex processing, hydraulic oil pollution and high cost make the system inefficiency.

Screw transmission: It adapts to the occasion in which long time continuous work and high working speed are needed. The advantage of Screw drive with simple structure is that it can transform rotary movement into linear motion with higher motion accuracy and smooth process. It is self-locking and of easy fabrication, low noise and can bear greater axial force. But the inefficiency of screw drive and the relative motion surface wear make it not an optimal device.

Chain transmission: Chain is mainly applied to the occasion that requires accurate transmission ratio requirement and far distance of shaft centres. The structure of chain drive is compact with high transmission efficiency and high accuracy. It is combined with no sliding and can be used in high temperature as well as high humidity environment. It can transfer power even when the distance of shaft centre is farther. The role of the shaft load is smaller and does not need a lot of tension force. The disadvantage of chain drive is that it can only be used for parallel axial transmission and its instantaneous velocity varied. The smoothness of chain drive is less than belt transmission. It should not be applied to transmission with large load change and transient reverse. 


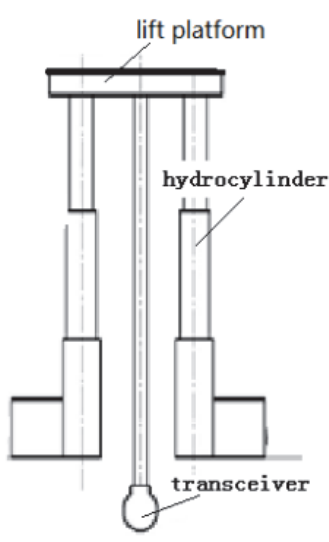

(a)

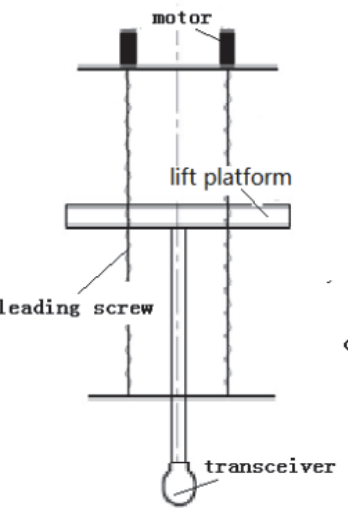

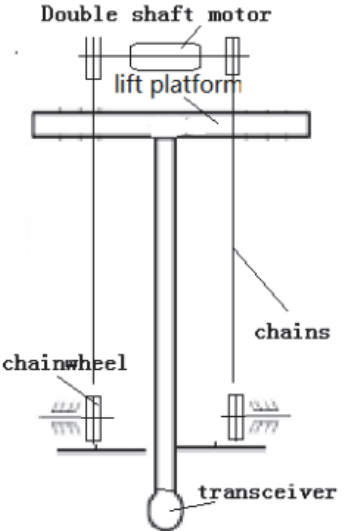

(c)

Figure 1 Design scheme: (a) hydraulic transmission; (b) Screw transmission; (c) chain transmission

\subsection{Design Scheme Comparison}

During the hydraulic transmission, hydraulic oil leakage and liquid compressibility can affect the accuracy of the executive element movement, which cannot meet the requirements of the repositioning precision device [6]. In addition, the traction efficiency of hydraulic transmission is also poor. It is unable to support the engine and achieve manual emergency retracting function to meet the application requirements when there are no special devices. It is required to use duplex multistage hydraulic drive. This makes the entire unit too big in weight and size and unable to be installed on the ship.

Screw drive is too rigid to adapt the ocean environment with alternating load and larger swaying. Due to the sever wear of contact surface and marine corrosion, the accuracy of device cannot be maintained easily. In addition, it is difficult to achieve two synchronous rotations of motor installed in the transducer when using screw drive.

The operating requirement of the device is reliable, low speed and heavy loading. The transmission distance of this device is $l(l=3000 \mathrm{~mm})$, which belongs to the remote transmission. The whole structure is compact with higher manufacturing and installation accuracy and requires working in a humid environment. In view of these factors, chain transmission will be the best choice. It can meet the design requirements and simplify the device structure with lighter weight.

\subsection{Basic Structure}

The structure of the USBL platform is shown in Fig. 2. The bracket is the main support structure. The motor which is the power unit of the lifting platform is arranged on the upper end of the bracket and uses the program to control time cycles and movement. The bearing seat is arranged on the bottom end of the bracket aiming to seal and support transducer axis. The motor, the bracket and the transducer shaft are connected by chain transmission, thus the transducer axis can be driven through the rotation of the motor moving up and down. The vertical bracket is equipped with two parallel linear guides. There is a hand wheel on the motor, which can drive the transducer axis to the target position in case of power down. The repairing box makes transducer easier for installation and maintenance. Through-holes of DN500 are designed on the repair box and sea gate which meet the requirements of the diameter of the transducer $\left(D_{0}=400 \mathrm{~mm}\right)$. The repair box is $650 \mathrm{~mm}$ long, $650 \mathrm{~mm}$ wide, and $600 \mathrm{~mm}$ high, whose double-side door can be opened in order to facilitate maintenance of the transducer. When necessary, the transducer can be lifted to the repair box position from chain driving system. Then close the gate valves, relief internal water pressure, open the double-side door and remove the transducer. Under normal work conditions, the double-side door has to be well sealed. All the properties of repair box have been designed with reference to the parameters of the transducer.

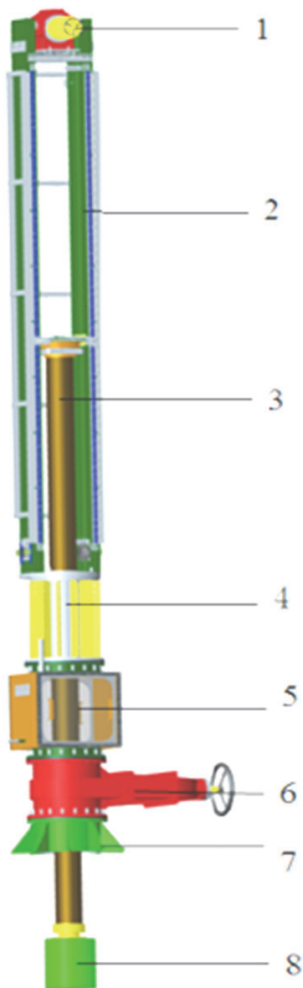

Figure 2 Assembly diagram of lifter: 1 - motor, 2 - bracket, 3 - transducer shaft, 4 - bearing seat, 5 - repair box, 6 - gate valve, 7 - pedestal, 8 - transducer

\section{THE KEY STRUCTURE ANALYSIS AND REALIZATION SCHEME}

\subsection{The Boundary Conditions of Theory Design}

This device can withstand bad working conditions such as level 4 waves [7]. The maximum ship roll swing is 
$15^{\circ}$ and cycle $6.5 \mathrm{~s}$. The transducer shaft protrudes from the bottom of the ship $3000 \mathrm{~mm}$, the relative velocity is 6 knots $(3.087 \mathrm{~m} / \mathrm{s})$ and the sea water resistance is $4215.4 \mathrm{~N}$ which is measured by the calculation of marishon method. The overall weight of the transducer and connection part is $0.965 \mathrm{t}$ and water resistance (profile resistance namely type resistance) is proportional to the sum of friction and differential pressure force.

$F_{1}=C_{D}\left(\frac{1}{2} \rho v^{2}\right) A_{P}$

where: $C_{D}$ type resistance coefficient $=1.0$.
$A_{P}$ the upstream area $0.21 \cdot 2.75+0.25 \cdot 0.35=0.665 \mathrm{~m}^{2}$. $\rho$ seawater density $=1.03 \times 10^{3} \mathrm{~kg} / \mathrm{m}^{3}$.

$v_{1}=0.514 \cdot 6=3.084 \mathrm{~m} / \mathrm{s}$.

$v_{2}=$ ship's side wave velocity

$v_{2}=\frac{(7.5+3) \cdot \sin 15 \cdot 4}{6.5}=1.6723 \mathrm{~m} / \mathrm{s}$.

$v=$ maximum speed of vertical rod end

$v=\sqrt{v_{1}^{2}+v_{2}^{2}}=3.51089 \mathrm{~m} / \mathrm{s}$.

Thus the water resistance force can be calculated by Eq. (1).

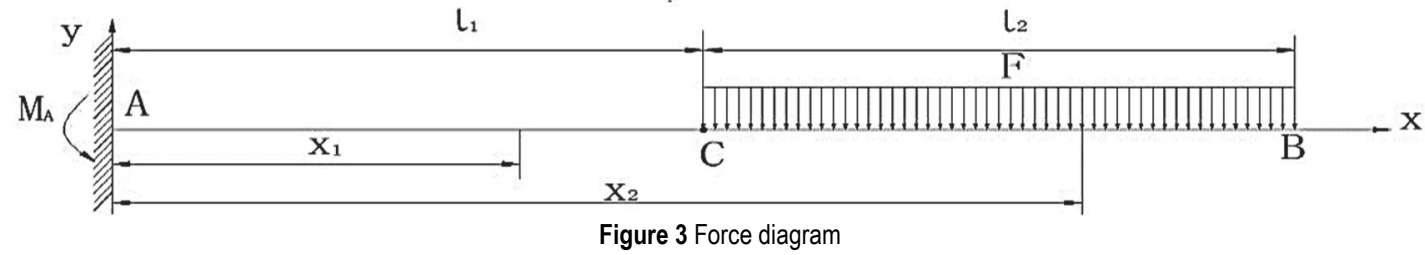

\subsection{Theoretical Calculation of the Transducer Axis}

The total length of transducer axis and its accessory structures is $L=5875 \mathrm{~mm}$. According to the requirement that the deflection angle is less than $0.1^{\circ}$, the distance depart from the center axis at the end of the transducer is $w$.

$$
w=L \cdot \tan \theta=5875 \cdot 0.00175=10.25 \mathrm{~mm}
$$

Thus the total deformation of transducer shaft should be less than $10.25 \mathrm{~mm}$ when actually working.

For the request rigid of the transducer axis is higher, 2205 duplex stainless steel has been used as the transducer shaft material. 2205 duplex stainless steel has favorable comprehensive performance: low temperature resistance, good toughness, high strength, fatigue and stress corrosion crack insensitive, etc. And it is widely used in pipe, boiler, shipbuilding and other fields with excellent formability of welding $[8,9]$.

Considering the most dangerous working conditions that the transducer extends out of the bottom of the ship 3 $\mathrm{m}$, the science model can be simplified as shown in Fig. 3 $[10,11]$ : the upper end of the transducer shaft is fixed, and uniformly distributed load by $4215.1 \mathrm{~N}$ is within a range of $3 \mathrm{~m}$. The detailed calculation process is presented as follows:

Coordinated system has been selected as shown in Fig. 3. Moment equation, the deflection line differential equation and integral equation:

For AC:

$$
\left\{\begin{array}{c}
M\left(x_{1}\right)=-q l_{2}\left(l_{1}+\frac{l_{2}}{2}-x_{1}\right) \quad\left(0 \leq x_{1} \leq l_{1}\right) \\
E I w_{1}=-q \frac{l_{2}}{6}\left(l_{1}+\frac{l_{2}}{2}-x_{1}\right)^{3}+C_{1} x_{1}+D_{1}
\end{array}\right.
$$

For CB:

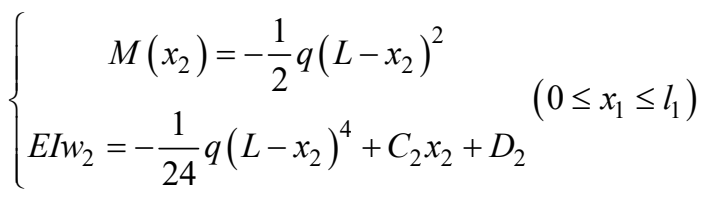

Then the integration constant can be established according to the boundary conditions and the continuity conditions:

$l_{1}=2.875 \mathrm{~m}$

$l_{1}=2.875 \mathrm{~m}$

$l_{1}=2.875 \mathrm{~m}$

$l_{1}=2.875 \mathrm{~m}$

Deflection line equation and angle equation for each segment are presented:

$l_{1}=2.875 \mathrm{~m}$

$l_{1}=2.875 \mathrm{~m}$

$l_{1}=2.875 \mathrm{~m}$

$l_{1}=2.875 \mathrm{~m}$

Then deflection line equation and angle equation of free end can be derived:

$l_{1}=2.875 \mathrm{~m}$

$l_{1}=2.875 \mathrm{~m}$

where:

$$
\begin{aligned}
& q=\frac{F}{l_{2}}=\frac{4512.4 \mathrm{~N}}{3 \mathrm{~m}}=1504.13 \mathrm{~N} / \mathrm{m} \\
& l_{1}=2.875 \mathrm{~m} \\
& l_{1}=2.875 \mathrm{~m} \\
& E=200 \mathrm{GPa} \\
& d=150 \mathrm{~mm} \\
& D=210 \mathrm{~mm}
\end{aligned}
$$


$I=\frac{\pi}{64}\left(D^{4}-d^{4}\right)=7.057935 \times 10^{-5} \mathrm{~m}^{4}$

Then $w_{B}$ and $\theta_{B}$ are figured out:

$$
\begin{aligned}
& w_{B}=\left.w_{2}\left(x_{2}\right)\right|_{x_{2}=L}=9.051 \mathrm{~mm} \\
& \theta_{B}=\left.w_{2}^{\prime}\left(x_{2}\right)\right|_{x_{2}=L}=0.0134^{\circ}
\end{aligned}
$$

\subsection{Finite Element Analysis of the Transducer Axis}

Using finite element analysis software ANSYS analyses transducer axis and the simulation analysis process is shown as follows. Fig. 4 shows a limited case of a boundary condition where two sides of the bearing have been fixed. At 3 meters of the bottom shaft is applied uniformly distributed force by $4215.4 \mathrm{~N}$ on half shaft surface. Fig. 5 shows the analysis results of total deformation and equivalent stress.

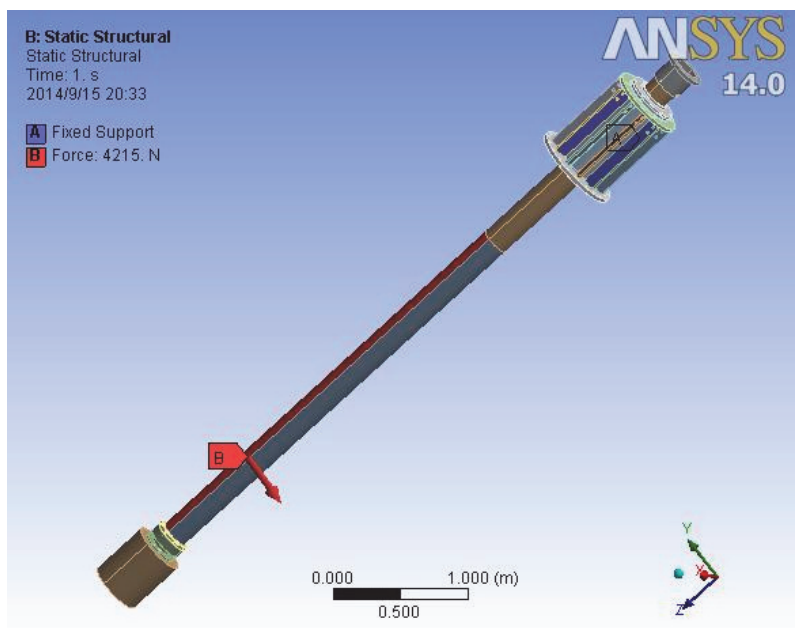

Figure 4 Boundary limited conditions

The results of the analysis show that the maximum deflection displacement of the end is $4.21 \mathrm{~mm}$ and the maximum stress is $80.4 \mathrm{MPa}$ with the safety factor of 2,8 . It complies with the design requirements.

\section{TESTS}

\subsection{A Description of the Test Program}

Performance testing: first, we developed a set of suitable bracket and then mounted the part that is above repairing box of lifting device integrally on the bracket. Equivalent load was applied to the bracket (see Fig. 6). Then the electrical control system and the precision of lifting position were tested.

\subsection{A Description of the Test Process}

(1) During normal operation of the apparatus, a stopwatch meter was used to measure the speed of operation. Each run $3 \mathrm{~m}$ as one test and repeated 10 times each test. Then the average running time was obtained as a record node and totally run 16 records node, which means the upper and down run 160 times.

(2) We used a laser device to measure the position accuracy. A lateral load of $366 \mathrm{~kg}$ was applied at a distance of $1.25 \mathrm{~m}$ of the bottom shell plate and at the end of the shaft was applied $54 \mathrm{~kg}$ load to simulate resistance of seawater. Each ran 10 times and the average localization error and offset were calculated as one record node and totally ran 16 records node.

(3) The test of sealed bearings: we used intracavity hydraulic method to make equivalent measurement and the test pressure was $0.2 \mathrm{MPa}$. Experiments had been maintained for 15 minutes. 16 group tests were carried out and each test interval lasted more than 30 minutes.

\subsection{The Analysis of Experimental Results}

The result of the tests showed that the device overall ran smoothly and the electrical control system worked stably. The lifter ran up and down at the speed of $0.066 \mathrm{~m} / \mathrm{s}$ and the margin of localization error was within $0.2 \mathrm{~mm}$. The deflection angle was less than $0.02^{\circ}$. The average end offset was less than $6.5 \mathrm{~mm}$ when with load while $0 \mathrm{~mm}$ without load. All the data conformed to the design requirements.

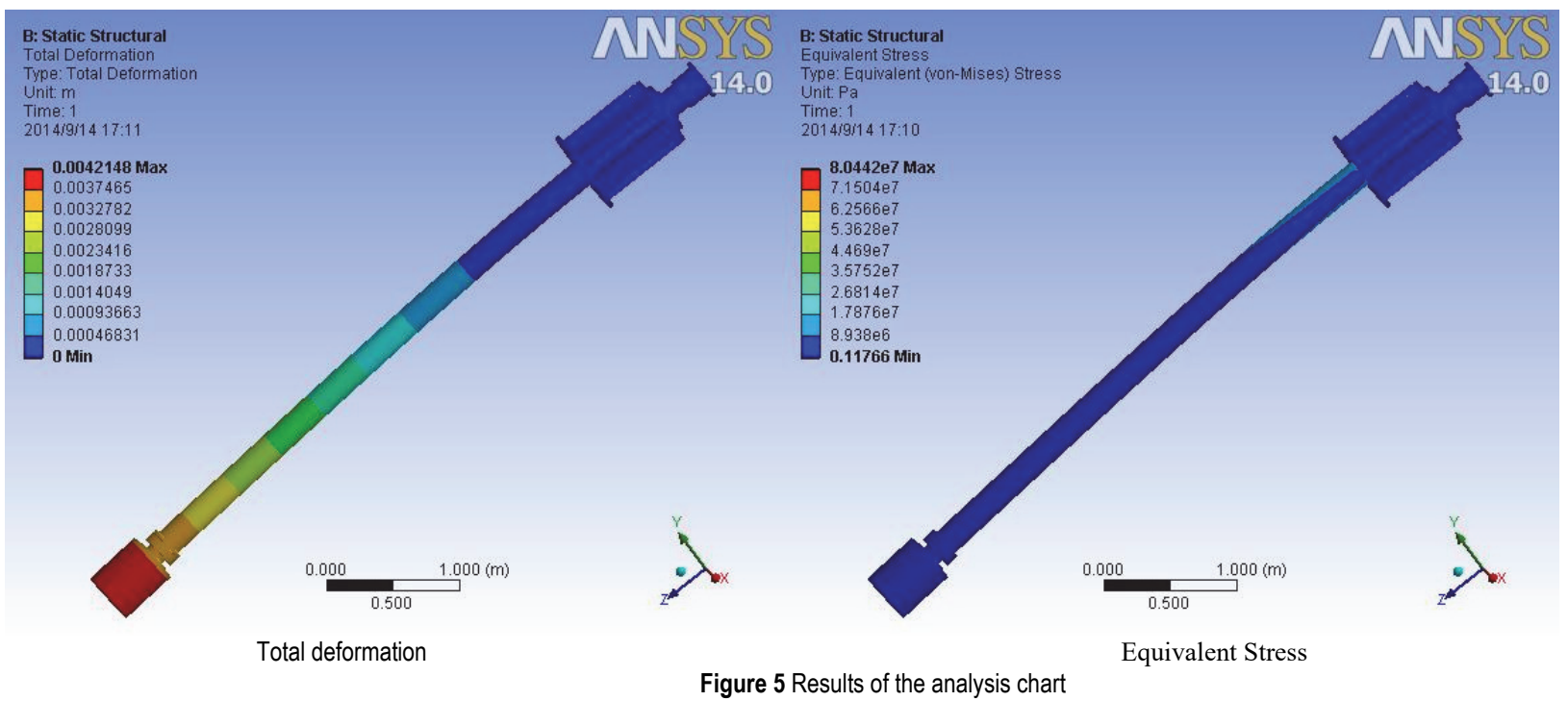




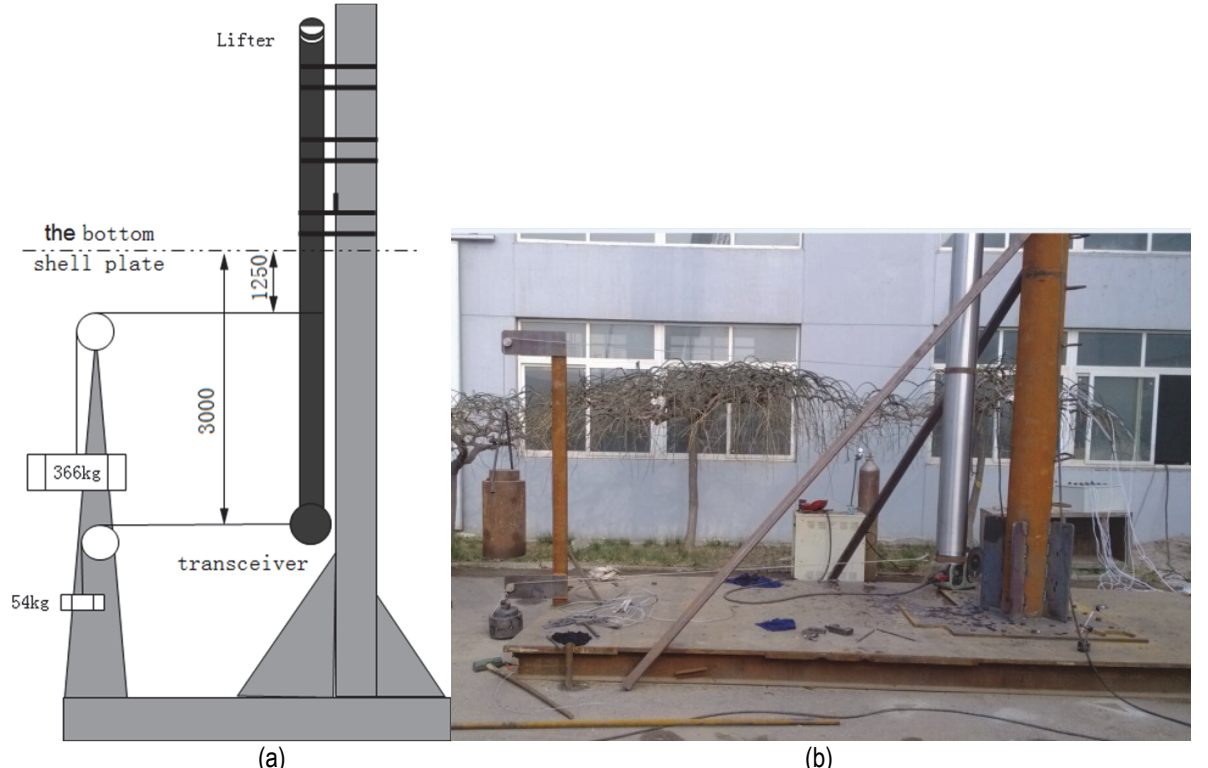

Figure 6 Offshore test: (a) Offshore testing program; (b) Offshore testing photo

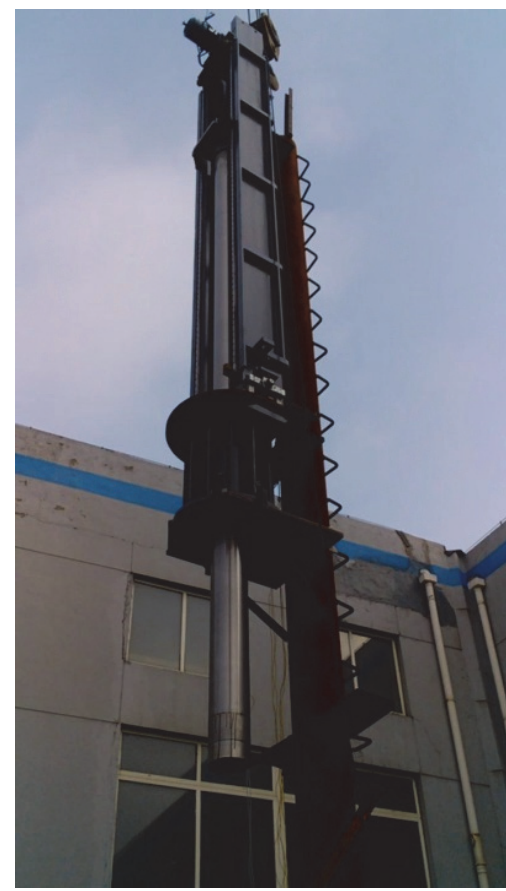

Figure 7 End offset measurement photo

Table 1 Recording test table of a device function

\begin{tabular}{|c|c|c|}
\hline Project & $\begin{array}{c}\text { Record } \\
\text { (normal) }\end{array}$ & Remark \\
\hline $\begin{array}{c}\text { The integrity of the device design } \\
\text { and processing components }\end{array}$ & normal & $\begin{array}{c}\text { After assembly is } \\
\text { completed test }\end{array}$ \\
\hline The smooth running of the device & normal & Inching automatic \\
\hline The tightness of the bearing & normal & $\begin{array}{c}\text { Spills phenomenon did } \\
\text { not occur during the } \\
\text { experiment }\end{array}$ \\
\hline
\end{tabular}

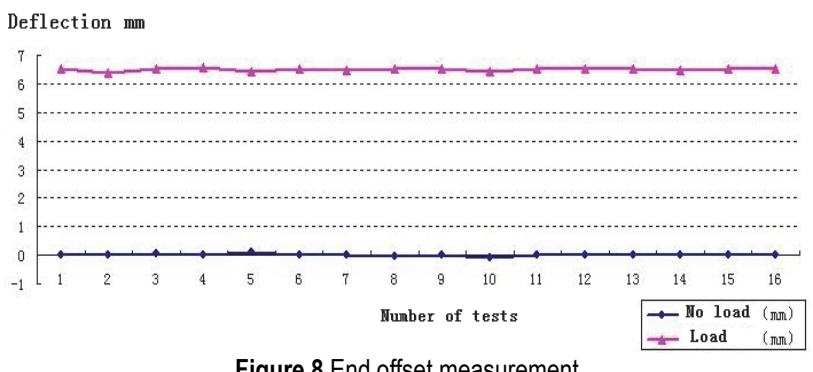

Figure 8 End offset measurement

\section{CONCLUSIONS}

In this paper we have designed a guide lifter for the transceiver of USBL. Through the testing and validation, the device can stably run under level 4 waves conditions. It also can ensure the positioning accuracy in $0,2 \mathrm{~mm}$ and the deflection angle is less than $0.02^{\circ}$ when the transceiver extends $3000 \mathrm{~mm}$ out the bottom of the ship. This has reached the design parameters and become the leading position worldwide. And this device has been affirmed by the user as a completely independent property rights in China's first USBL lifting device.

\section{Acknowledgements}

This paper was supported in part by the Key R\&D Program of Shandong Province in 2019 (Public Welfare Science and Technology Tackling Category) (2019GGX104071), Yantai Science and Technology Innovation Development Project (2020XDRH094), Industry-university-research Foundation project of Yantai Institute of Information Technology of Shanghai Jiao Tong University (G19YTJC013, G19YTJC012), and Doctoral Program of Shandong Provincial Natural Science Foundation of China (ZR2019BF048).

\section{REFERENCES}

[1] Sea Technology Group (2012). ARA Deploys Sonardyne USBL System in Port Survey. Sea Technology, 53(8), 59.

[2] Morgado, M., Batista, P., Oliveira, P., \& Silvestre, C. (2011). Position USBL/DVL sensor-based navigation filter in the presence of unknown ocean currents. Automatica, 47(12), 2604-2614. https://doi.org/10.1016/j.automatica.2011.09.024

[3] Li, J. M., Yao, Z. G., Zhang, W. H. et al. (2009). Development of Ultra-short baseline extensometer. Journal of Geodesy and Geodynamics, 29(4), 144-147.

[4] Arkhipov, M. (2009). An Approach for Design of Multielement USBL Systems. WSEAS Transactions on Systems, 8(8), 957-567.

[5] Liu, C. (2013). Design and research of USBL. YanTai University. 
[6] Min, Y. \& Junyin, H. (2010). The calibration of the USBL transducer array for Long-range precision underwater positioning. The 10th IEEE International Conference on Signal Processing (ICSP 2010), 2357-2360. https://doi.org/10.1109/ICOSP.2010.5657193

[7] Sun, C. G., Gong, S. G., \& Ren, D. K. (2008). Measurement experiment study on ocean wave pressure field under rough sea conditions. Chinese Journal of Scientific Instrument, 29(7), 1503-1506.

[8] Zhang, Z. H. (2004). The test of dot taint for 2205 stainless steel. Machine Building \& Automation, 33(4), 57-58.

[9] Wang, Z. X., Zhang, X., \& Zhang, J. X. (2011). Fatigue crack behavior of welded structure of duplex stainless steel 2005. Journal of Chongqing Jiaotong University, 30(4), 880884.

[10] Wang, H. X. (1996). Periscope damper and turning point stable structure. Ships \& Optics, 3, 31-36.

[11] Wang, Y. F. \& Chen, W. Y. (2010). Analysis of excitation response caused by the periscope drawtube abstract. Ship Electronic Engineering, 1, 194-200.

\section{Contact information:}

Chai YONGSHENG, Assistant Professor

Yantai University,

School of Mechatronics and Automobile Engineering,

32 Qingquan RD Laishan District, Yantai, China

E-mail: chaiysh@163.com

Min XIAOCHEN, postgraduate

Yantai University,

School of Mechatronics and Automobile Engineering,

32 Qingquan RD Laishan District, Yantai, China

E-mail: 527932779@qq.com

Liu MI, postgraduate

Yantai University,

School of Mechatronics and Automobile Engineering,

32 Qingquan RD Laishan District, Yantai, China

E-mail:17862817911@163.com

Zhang MAOYUAN, postgraduate

Yantai University,

School of Mechatronics and Automobile Engineering,

32 Qingquan RD Laishan District, Yantai, China

E-mail:1019700971@qq.com

Wei SHANGTAO, postgraduate

Yantai University,

School of Mechatronics and Automobile Engineering,

32 Qingquan RD Laishan District, Yantai, China

E-mail:1157168563@qq.com

\section{Wang CHANGHUI, lectorate}

(Corresponding author)

Yantai University,

School of Mechatronics and Automobile Engineering,

32 Qingquan RD Laishan District, Yantai, China

E-mail: wang_changhui@ytu.edu.cn

\section{Liang MEl, lectorate}

Yantai University,

School of Mechatronics and Automobile Engineering,

32 Qingquan RD Laishan District, Yantai, China

E-mail: mmglm@163.com 